\title{
In utero supplementation with methyl donors enhances allergic airway disease in mice
}

\author{
John W. Hollingsworth,1,2 Shuichiro Maruoka, ${ }^{2}$ Kathy Boon, ${ }^{2,3}$ Stavros Garantziotis, ${ }^{1,2,4}$ \\ Zhuowei Li, ${ }^{1,2}$ John Tomfohr, ${ }^{2}$ Nathaniel Bailey, ${ }^{2}$ Erin N. Potts, ${ }^{1}$ Gregory Whitehead, ${ }^{4}$ \\ David M. Brass, ${ }^{2,5}$ and David A. Schwartz ${ }^{2,6}$
}

\begin{abstract}
1Division of Pulmonary, Allergy and Critical Care Medicine, Department of Medicine, Duke University Medical Center, Durham, North Carolina, USA. 2Environmental Lung Disease Laboratory, National Heart Lung and Blood Institute, Research Triangle Park, North Carolina, USA. ${ }^{30}$ fffice of Cancer Genomics, National Cancer Institute, Bethesda, Maryland, USA. ${ }^{4}$ Laboratory of Respiratory Biology, National Institute of Environmental Health Sciences, Research Triangle Park, North Carolina, USA. ${ }^{5}$ Neonatal-Perinatal Research Institute, Duke University Medical Center, Durham, North Carolina, USA. ${ }^{6}$ Division of Pulmonary and Critical Care Medicine, Department of Medicine, National Jewish Health, Denver, Colorado, USA.
\end{abstract}

\begin{abstract}
Asthma is a complex heritable disease that is increasing in prevalence and severity, particularly in developed countries such as the United States, where $11 \%$ of the population is affected. The contribution of environmental and genetic factors to this growing epidemic is currently not well understood. We developed the hypothesis, based on previous literature, that changes in DNA methylation resulting in aberrant gene transcription may enhance the risk of developing allergic airway disease. Our findings indicate that in mice, a maternal diet supplemented with methyl donors enhanced the severity of allergic airway disease that was inherited transgenerationally. Using a genomic approach, we discovered 82 gene-associated loci that were differentially methylated after in utero supplementation with a methyl-rich diet. These methylation changes were associated with decreased transcriptional activity and increased disease severity. Runt-related transcription factor 3 (Runx3), a gene known to negatively regulate allergic airway disease, was found to be excessively methylated, and $R u n x 3$ mRNA and protein levels were suppressed in progeny exposed in utero to a high-methylation diet. Moreover, treatment with a demethylating agent increased $R u n x 3$ gene transcription, further supporting our claim that a methyl-rich diet can affect methylation status and consequent transcriptional regulation. Our findings indicate that dietary factors can modify the heritable risk of allergic airway disease through epigenetic mechanisms during a vulnerable period of fetal development in mice.
\end{abstract}

\section{Introduction}

Asthma is a complex heritable disease that is increasing in severity and prevalence, particularly in developed countries. Currently, asthma affects $11 \%$ of the US population and accounts for $\$ 9.4$ billion of direct health care costs (1). However, the combination of genetic and environmental factors that contributes to this growing epidemic of allergic asthma is not well understood. While unique inhaled exposures likely contribute to the development of asthma (2), other environmental exposures, such as dietary changes, could also be contributing to these changing patterns of disease.

Pregnant women in developed countries now have diets supplemented with folate, designed to protect the fetus from developing facial clefting as well as neural tube and cardiac defects (3). Importantly, this prenatal supplement is a source of methyl donors, which could alter DNA methylation and may affect genetic expression $(4,5)$. Methylation of $\mathrm{CPG}$ islands within regulatory regions of DNA is an important mechanism to control transcriptional activity (5). The level of CPG methylation within specific genetic regions appears transmissible across generations (6). In fact, it has been shown that the concentration of methyl donors in the diet of pregnant agouti mice has transgenerational effects on coat color of the progeny due to altered expression of the agouti gene resulting from

Nonstandard abbreviations used: HMD, high-methyl donor diet; LMD, lowmethyl donor diet; MSDK, methylation-specific digital karyotyping; RD, regular diet; Runx3, runt-related transcription factor 3.

Conflict of interest: The authors have declared that no conflict of interest exists. Citation for this article: J. Clin. Invest. 118:3462-3469 (2008). doi:10.1172/JCI34378. methylation of the agouti promoter $(7,8)$. Emerging research indicates that epigenetic mechanisms affect the expression of transcription factors that control the lineage of Th1 (9-11), Th2 (11-13), and Treg (14) cells. For instance, hypomethylation of naive T cells enhances the expression of FoxP3 $(14,15)$ and STAT4 $(9-11)$, resulting in the expansion of Treg and Th1 cells, supporting the hypothesis that hypomethylation could prevent allergic airway disease by increasing the population of Treg cells and skewing toward a Th1 phenotype. Based on these observations, we hypothesized that in utero exposure to a diet rich in methyl donors could reduce the expression of key genes that regulate the development of an adaptive immune response, increasing Th2 immunity and enhancing the heritable risk of developing allergic airway disease.

To pursue this hypothesis, we evaluated the effects of in utero dietary modifications on the severity of allergic airway disease in mice and on site-specific DNA methylation throughout the genome. We report that maternal dietary intake of methyl donors enhances the severity of allergic airway disease in offspring of C57BL/6J mice and that this trait is inherited transgenerationally. We further show that in utero dietary supplementation with methyl donors can enhance DNA methylation at specific CPG motifs, which is associated with both altered gene expression and the severity of allergic airway disease. Using a demethylating agent, we demonstrated transcriptional regulation through DNA methylation. Our findings indicate that in a mouse model in utero, dietary factors can modify the heritable risk of allergic airway disease during a vulnerable period of fetal development through epigenetic mechanisms. 
A
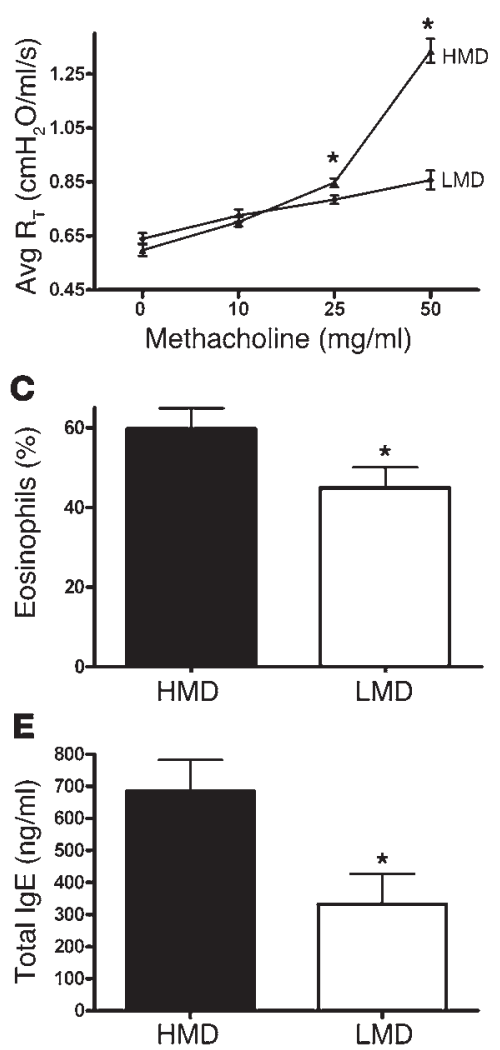

G

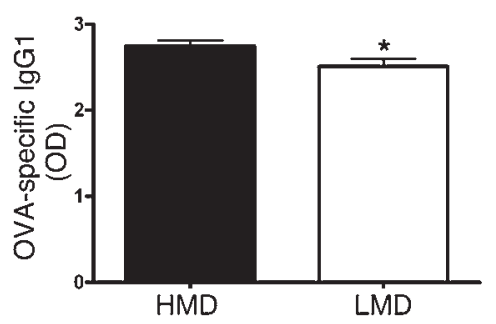

\section{B}

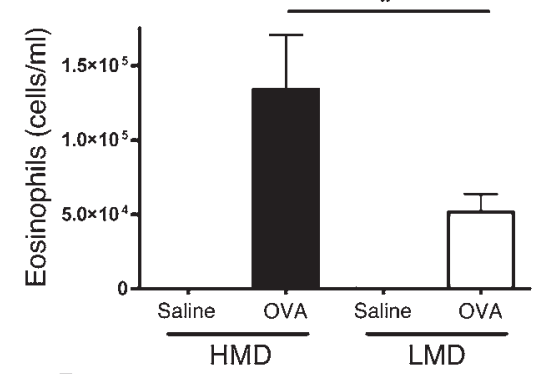

D
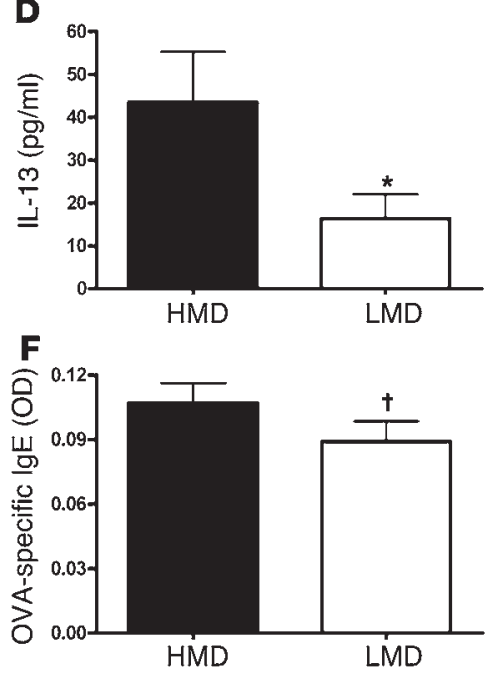

Figure 1

Gestational exposure to dietary methyl donors regulates severity of allergic asthma. Adult C57BL/6J progeny exposed to HMD in utero demonstrate enhanced allergic inflammation when compared with those exposed to LMD. C57BL/6J breeding pairs were fed HMD, RD (NIH-31), or LMD beginning 2 weeks prior to mating and during pregnancy. Experimental diets were continued until the time of weaning, when $\mathrm{F} 1$ progeny were placed on $\mathrm{RD}$ (NIH-31). At 6 to 10 weeks of age, F1 mice were immunized and subjected to an OVA challenge. In utero exposure to HMD was associated with enhanced (A) airway responsiveness to methacholine $(n=5-6$; ${ }^{\star} P<0.05$ ) (HMD, squares; LMD, diamonds), (B) concentration of lung lavage eosinophils ( $n=31-63$; HMD, white bars; LMD, black bars), (C) percentage of lung lavage eosinophils $(n=31-63)$, (D) IL-13 levels in whole-lung lavage fluid $(n=15-26)$, (E) total serum IgE ( $n=15-26$; ${ }^{*} P<0.05$, HMD versus LMD), (F) OVA-specific $\operatorname{lgE}\left(n=15-26 ;{ }^{\dagger} P=0.05, \mathrm{HMD}\right.$ versus $\left.\mathrm{LMD}\right)$, and (G) OVA-specific IgG1 ( $n=15-26$; ${ }^{*} P<0.05$, HMD versus LMD). Avg, average.

\section{Results}

In utero exposure to dietary methyl donors affects the severity of airway disease. We first compared the development of allergic airway disease in the progeny of $\mathrm{C} 57 \mathrm{BL} / 6 \mathrm{~J}$ dams exposed to diets that were supplemented with reduced or enhanced levels of methyl donors (Supplemental Table 1; supplemental material available online with this article; doi:10.1172/JCI34378DS1). In the initial experiments, dams and pups were maintained on respective diets throughout the experimental protocol. The F1 progeny exposed to an in utero and postweaning diet supplemented with methyl donors demonstrated enhanced cardinal features of allergic airway disease, including airway hyperreactivity, lung lavage eosinophilia and IL-13, and higher concentrations of serum IgE (Supplemental Figure 1). Although these observations support the role of diet in altering the development and severity of allergic airway disease, the impact of in utero versus postweaning dietary exposure to methyl donors remained unclear. For this reason, we exposed dams to the experimental diets and pups were placed on regular diets (RDs) at the time of weaning. Under these conditions, in utero exposure to a diet supplemented with a high concentration of methyl donors enhanced the devel- opment and severity of allergic airway disease (increased airway hyperreactivity, lung lavage eosinophilia and IL-13, higher concentrations of serum IgE, OVA-specific IgE, and OVA-specific IgG and histologic evidence of allergic airway disease) in the F1 progeny (Figures 1 and 2). Animals exposed to in utero high-methyl donor diet (HMD) (versus low-methyl donor diet [LMD]) demonstrated no significant difference in IgG2a (data not shown). The mice gestated on an RD demonstrated an intermediate phenotype (Supplemental Figure 2). Although somewhat less robust, the effect of in utero dietary modification on the allergic airway phenotype was paternally transmitted to the F2 generation with more eosinophilic airway inflammation and higher concentrations of total serum IgE from male F1 mice exposed in utero to an HMD (Figure 3). In contrast to in utero exposure, dietary methyl donor exposure solely during either lactation or adulthood (between 3 and 10 weeks after gestation) did not result in significant differences in the severity of allergic airway disease (Supplemental Figures 3 and 4). These observations indicate that gestation represents a unique period of enhanced vulnerability to methyl donors that can predispose offspring to the future development of allergic airway disease. 


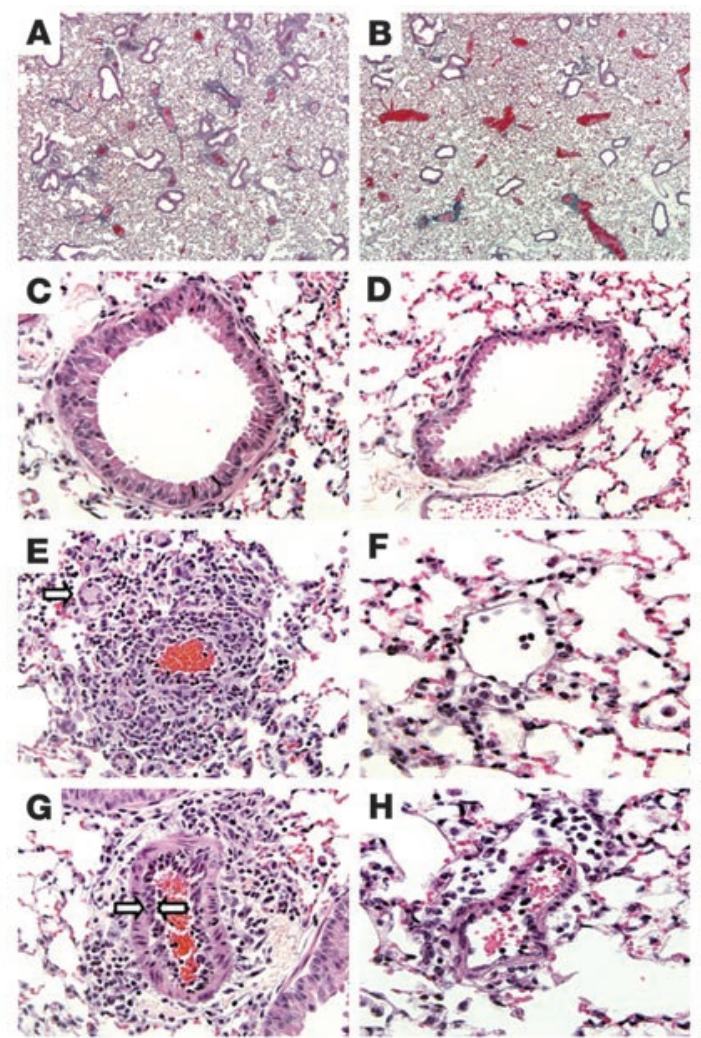

In utero exposure to dietary methyl donors alters lymphocyte function. Next, we observed that in utero exposure to HMD was associated with increased ratio of $\mathrm{CD}^{+} / \mathrm{CD}^{+}$lymphocytes in the spleen (Figure 4A). This observation indicates that in utero exposures can affect $\mathrm{T}$ lymphocyte maturation and development. To determine whether in utero diet affected lymphocyte function, we harvested and cultured thymocytes, splenocytes, and mediastinal lymph nodes from immunized and OVA-challenged animals that were exposed in utero to either an HMD or an LMD. Although no consistent differences in Th2 cytokines (IL-4, IL-5, or IL-13) in the supernatant of cell cultures following exposure to OVA were detected, we identified robust differences in the CC chemokines KC, CCL4 (MIP1 $\beta$ ), and CCL5 (RANTES) derived from splenocytes, lymph nodes, and thymocytes (Figure 4B). These CC chemokines play an important role in chemotaxis

\section{Figure 2}

Gestational exposure to methyl donors enhances allergic airway disease in mice. H\&E staining of lung sections obtained from mice subjected in utero to HMD or LMD diet, then immunized and subjected to an OVA challenge. Low magnification of lungs from HMD (A) and LMD (B) mice. Higher magnification of small airways from HMD (C) and LMD (D) mice. Venule with perivascular cuffing from a dam fed HMD (E) or LMD (F) during gestation. Arrow shows multinucleated giant cells. Arteriole with perivascular cuffing from a dam fed HMD $(\mathbf{G})$ and $\mathrm{LMD}(\mathbf{H})$ during gestation. Arrows identify margination of inflammatory cells. Original magnification, $\times 4(\mathbf{A}$ and $B) ; \times 40(\mathbf{C}-\mathrm{H})$.

of monocytes and eosinophils and can contribute to the severity of allergic asthma (16).

To determine whether dietary exposure alone was sufficient to specifically modify $\mathrm{T}$ lymphocyte cytokine production, we isolated splenic $\mathrm{CD}^{+}$lymphocytes from mice gestated on either an HMD or LMD and stimulated them with monoclonal antibodies to $\mathrm{CD}^{+} \mathrm{CD}^{+} 8^{+}$. In this assay, we demonstrate that exposure to methyl donors enhances the production of IL-4 in $\mathrm{CD}^{+}$cells (Figure 4C). This finding suggests that in utero exposure to methyl donors favors lymphocyte maturation into a Th2 phenotype. In aggregate, these findings (Figures 1-4) indicate that in utero exposure to a diet rich in methyl donors enhances the development and severity of allergic airway disease by regulating differentiation of T lymphocytes and suggests that DNA methylation may play a role in the development of this phenotype.

The asthmatic phenotype is associated with enhanced site-specific $C p G$ methylation. To evaluate differences in DNA methylation, we analyzed both global methylation and site-specific methylation. We observed no significant differences in total DNA methylation of whole lung tissue between progeny of dams treated with high and low methylation diets as measured with a methylation-sensitive restriction enzyme assay. This was not surprising, since our LMD did contain the minimal concentration of methyl donors necessary to prevent congenital abnormalities. We subsequently reasoned that key regulatory regions affecting $\mathrm{T}$ cell maturation could skew toward a Th2 phenotype in the progeny of mice gestated on an HMD. To address this possibility, we measured methylation in 2-4 CpG sites within the promoter regions of the Th2 cytokines IL-4, IL-5, and IL-13 and a Th2 cytokine locus control region within rad50 (17), the RHS7 hypersensitivity site, and then used methylation-specific PCR to examine the methylation state of these CPG islands in lung tissue from our treatment groups. Importantly,
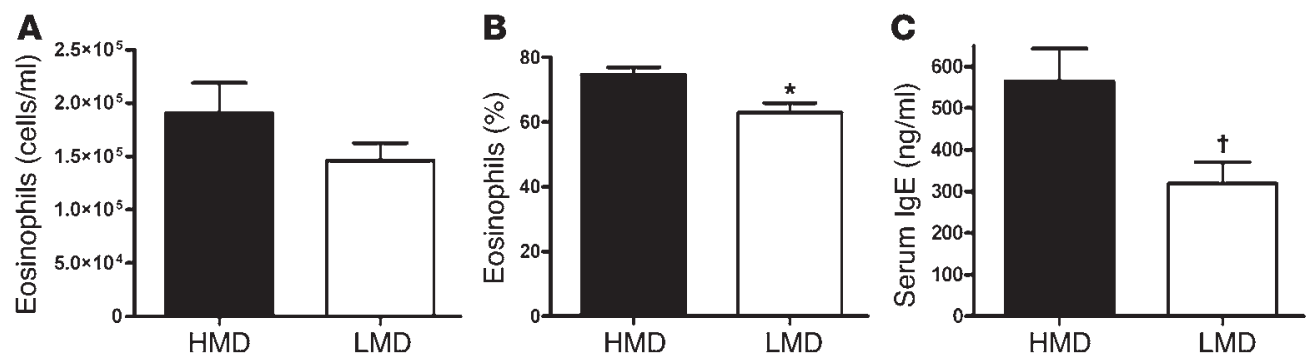

Figure 3

Transgenerational inheritance of diet-associated risk of allergic asthma. Male F1 progeny were mated with naive females and maintained on RD. F2 progeny were immunized and subjected to an OVA challenge at 6-10 weeks of age. The F2 progeny were kept on RD. F2 adults (HMD, $n=37$; LMD, $n=68$ ) demonstrated nonsignificant differences in the $(\mathbf{A})$ concentration of lung lavage eosinophils $(P=0.099)$, significant differences in the $(\mathbf{B})$ percentage of lung lavage eosinophils $\left({ }^{*} P=0.007\right),(\mathbf{C})$ and significant differences in serum $\lg \mathrm{E}\left({ }^{\dagger} P<0.001\right)$. 

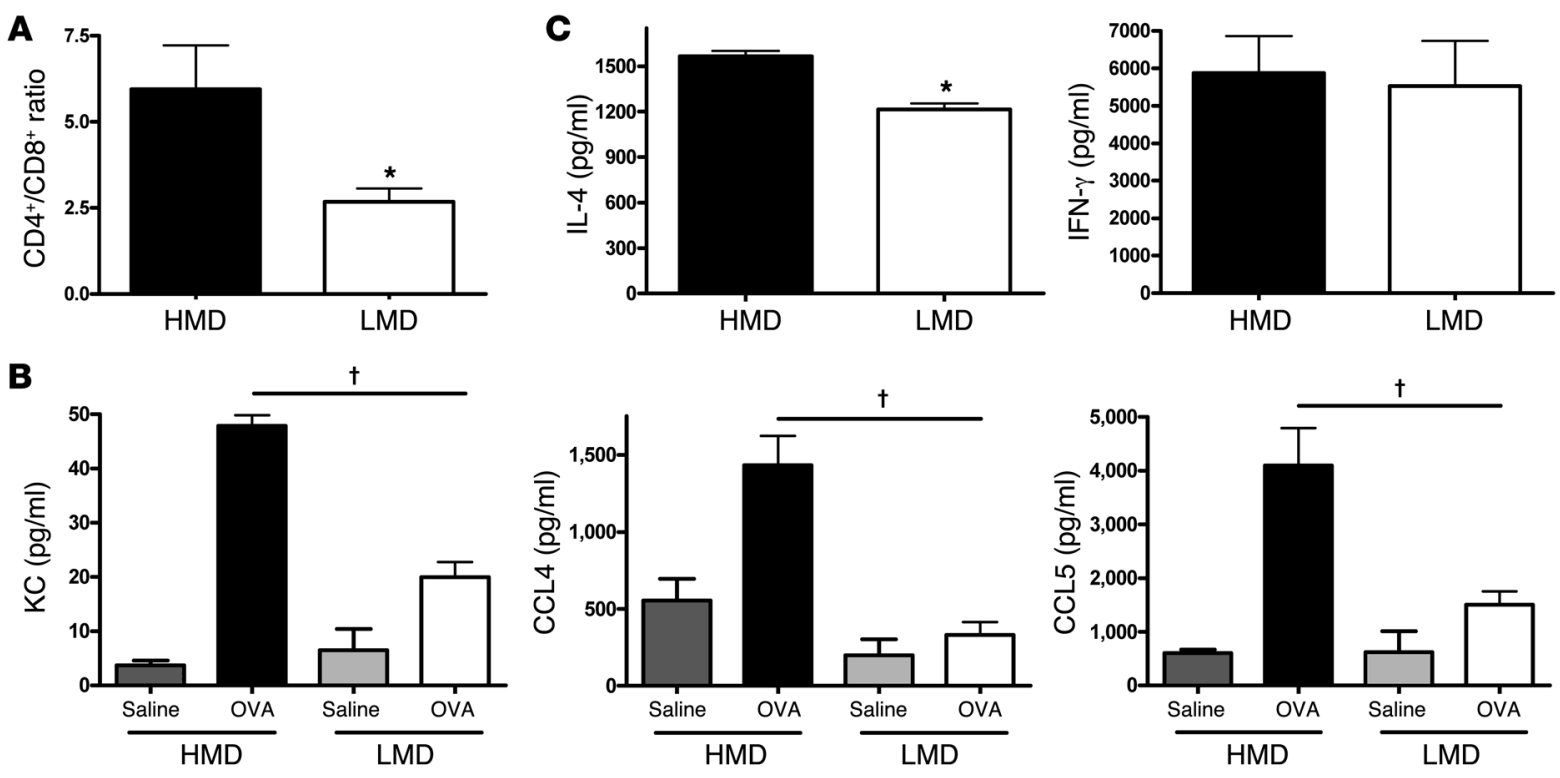

Figure 4

Diet modifies production of cytokines and chemokines in splenocytes. Spleens from mice gestated on either HMD or LMD were harvested following immunization and challenge with OVA. To determine functional consequences of perinatal diet, splenocytes were minced, passed through a $50-\mu \mathrm{m}$ cell strainer, and isolated by density gradient centrifugation with Histopaque 1083 as described in Methods. (A) CD4 $4^{+}$CD8 ${ }^{+}$ratios in the spleen were determined by flow cytometry $\left(n=5 ;{ }^{*} P<0.05\right.$, HMD versus LMD). To determine the functional implications of respective diets on splenocytes, $1 \times 10^{6}$ cells were exposed to saline or $4 \mu \mathrm{g}$ OVA in vitro for 72 hours. Supernatants were collected and evaluated for cytokines/ chemokines by Bio-Plex profiling. (B) Splenocytes derived from animals exposed to HMD demonstrate enhanced production of KC, CCL4, and CCL5 $\left({ }^{\dagger} P<0.01\right.$, HMD versus LMD). (C) Next, we harvested splenocytes from mice with gestational exposure to HMD or LMD without immunization and challenge with OVA and isolated CD4 ${ }^{+}$lymphocytes by negative selection. These cells were challenged with antibodies to $\mathrm{CD}^{+} \mathrm{CD}^{+} 8^{+}$ $(1 \mu \mathrm{g} / \mathrm{ml}$ each) for 48 hours. CD4+ cells from HMD mice demonstrate enhanced levels of IL-4 and no difference in IFN- $\gamma$.

this approach did not yield discernible differences between the mice gestated on either an HMD or an LMD and led us to speculate that key regulatory genes that controlled the development of an adaptive immune response were differentially methylated.

To screen for specific targets that were differentially methylated, we analyzed genome-wide site-specific DNA methylation in phenotypic extremes of our F1 progeny that were gestated on either an HMD or LMD. Using a modification of methylation-specific digital karyotyping (MSDK) (18), we profiled site-specific differences in DNA methylation throughout the genome in lung tissue from $\mathrm{F} 1$ mice with severe allergic airway disease (lavage eosinophils $>75 \%$ ) exposed in utero to an HMD and F1 mice with minimal allergic airway disease (lavage eosinophils $<25 \%$ ) exposed in utero to an LMD. To accomplish this, we generated 2 MSDK libraries and sequenced up to 70,000 genomic tags per library. After comparison of the 2 groups (severe allergic airway disease/HMD vs. minimal allergic airway disease/LMD), we selected for genomic tags with at least a 5-fold difference in tag counts between these groups. For the purposes of this investigation, we focused on the 121 loci that have lower genomic tag frequencies (higher level of methylation) in the asthmatic/HMD group. Of these 121 loci, 82 had a $P$ value of less than 0.05 (Supplemental Table 2). Conceptually, each of these loci represents a potential candidate gene that through altered expression may influence the risk of developing allergic airway disease.

The 10 most significantly differentially methylated genomic tags identified from the MSDK analysis represent regions of biological interest. These candidate regulatory regions were located in close proximity to the following genes: zinc finger DHHC domain containing $5(Z d h h c 5)$, very low-density lipoprotein receptor (Vldlr) sparc/osteonectin (Spock2), Cbp/p300 interacting transactivator (Cited4), cyclin M1 (Cnnm1), palmitoylated 5 (Mpp5), deoxyguanosine kinase (Dguok), $\alpha$-1,3-galactosyltransferase 2 (A3galt2), zincfinger protein NOLZ1 (Zfp503), and REST corepressor 3 (Rcor3). In addition, we identified several genes known to play a role in cellular migration (Rho GTPase [Rnd3] and CDC42 effector protein [Cdc42ep1]) and allergic airway disease (runt-related transcription factor 3 [Runx3], nuclear factor of activated T cells [Nfatc1], and Janus kinase 2 [Jak2]).

Validation of MSDK analysis. We analyzed differences in DNA methylation in regions of $\mathrm{CPG}$ islands located in close proximity to the genomic tag identified by MSDK and the transcription start site for 5 selected candidate genes (Runx3, Jak2, Nfatc1, Rcor3, Ube2j1) using base-specific cleavage and MALDI-TOF mass spectrometry. This method allowed us to measure the levels of DNA methylation in a quantitative manner revealing differential DNA methylation, though not all the $\mathrm{CpG}$ dinucleotides were informative in this analysis. Differential methylation within a predicted Pax2 transcription binding site (nucleotide 282 of CPG99 of Runx3) was associated with level of transcription. These results suggest that increased methylation correlates with reduced level of mRNA expression (Supplemental Figure 5).

To internally validate the results from the MSDK analysis, we measured the transcript levels of several candidate genes derived from the MSDK results in whole lung tissue from high responders/ HMD and low responders/LMD (Figure 5A). As expected, the hyper- 
A

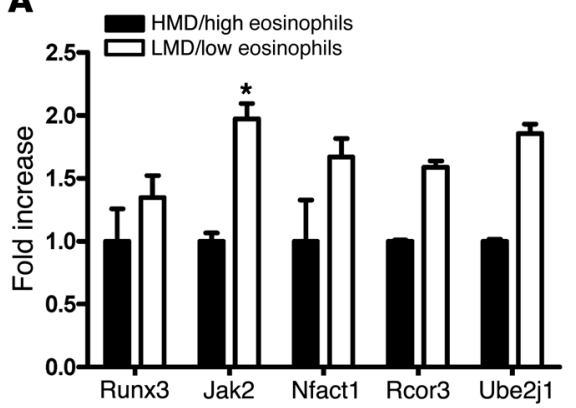

B
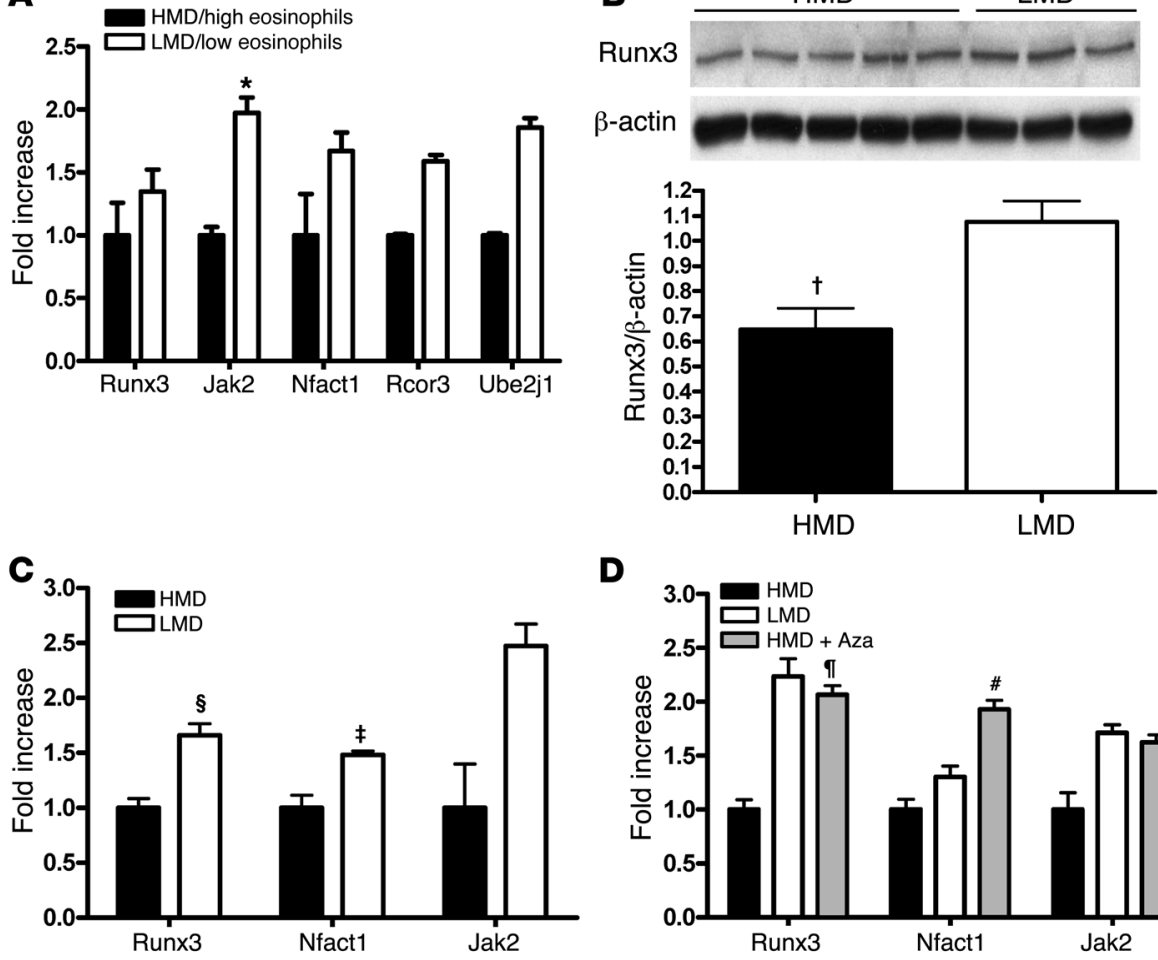

D

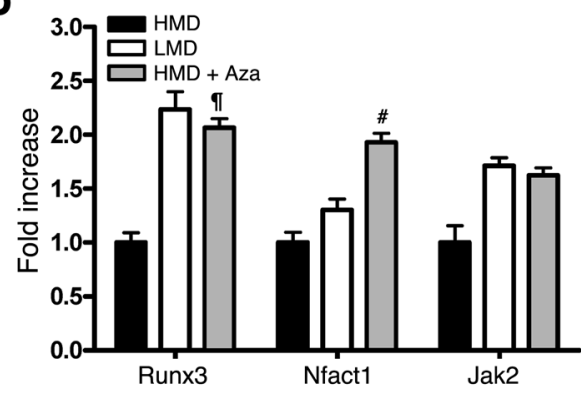

Figure 5

DNA methylation affects mRNA and protein expression. Relative mRNA expression was measured by real-time PCR using SYBR-Green as described in Methods. (A) Comparison between HMD/high responders $(n=8)$ and LMD/low responders $(n=3)$ in challenged C57BL/6 mice in lung tissue. $P=0.30$ (Runx3); $P=0.10$ (Nfact1); ${ }^{\star} P=0.02$ (Jak2); $P=0.20$ (Rcor3); $P=0.08$ (Ube2j1). (B) Runx3 protein expression was determined in lung tissue from individual F1 mice exposed in utero to either an HMD $(n=5)$ or an LMD $(n=3)$. Mouse Runx3 protein was detected in total lung cell extracts by a polyclonal antibody to human AML2-/Runx3. ${ }^{\dagger} P<0.05$, HMD versus LMD. (C) Comparison of gene expression from splenocytes obtained from F1 mice treated in utero with a high $(n=5)$ or low $(n=6)$ methylation diet and subsequently sensitized and subjected to an OVA challenge. $¥ P=0.01$ (Nfact1); $\$ P=0.03$ (Runx3); $P=0.06$ (Jak2). (D) In vitro effect of a demethylating agent on gene expression from splenocytes. Splenocytes were obtained from F1 mice treated in utero with an HMD following sensitization and challenge with OVA. $1 \times 10^{6}$ cells were exposed to $4 \mu \mathrm{g}$ OVA in vitro for 72 hours and either pulsed daily with saline or $1.0 \mu \mathrm{M}$ of azacytidine (Aza). IP $=0.003$ (Runx3); $P=0.008$ (Nfact1); ${ }^{*} P=0.08$ (Jak2).

methylated genes derived from the MSDK analysis (Runx3, Nfact1, Jak2, Rcor3, and Ube2j1) demonstrated reduced mRNA expression in lung tissue when we compared high responders/HMD to low responders/LMD (Figure 5A). To independently validate the MSDK analysis, we measured the concentration of Runx3 protein in lung tissue obtained from nonselected mice gestated on an HMD $(n=5)$ or LMD $(n=3)$ and found that mice treated in utero with an HMD had a significantly lower concentration of Runx3 protein in the lung tissue (Figure 5B). To further test the validity of these findings in a more homogeneous cell population, we isolated splenocytes from nonselected OVA-challenged mice exposed in utero to HMD or LMD. As shown in Figure 5C, the expression levels of Runx3 $(P=0.01)$, Nfatc1 $(P=0.03)$, and Jak2 $(P=0.06)$ are reduced in splenocytes obtained from HMD F1 mice.

5-Azacytidine reverses suppression of $m R N A$ expression. We reasoned that in vitro treatment with a demethylating agent would lead to an increased level of mRNA in splenocytes from mice treated in utero with an HMD. As shown in Figure 5D, there was an increase favor the future development of allergic diseases, such as asthma. Consistent with this logic, failure to suppress genes that negatively regulate $\mathrm{Th} 2$ and favor normal maturation of the immune system could lead to enhanced severity of allergic airway disease in adults. Our observation that exposure to methyl donors was associated with altered lymphocyte development and function suggests that suppression of regulatory genes might contribute to enhanced allergic airway disease observed in animals exposed to dietary methyl donors. Previous in vitro studies indicate that DNA methylation can regulate T lymphocyte differentiation to either Th1 or Th2 lineages $(9,11-14)$. In vitro, hypomethylation enhances the expression of STAT4 and IFN- $\gamma(11)$ in $\mathrm{CD}^{+} \mathrm{T}$ cells and induces FoxP3 expression (14), skewing toward a Th1 response and increasing the population of Treg cells. We provide in vivo evidence that in utero diet modifies differentiation of splenic $\mathrm{T}$ lymphocytes to express either $\mathrm{CD} 4$ or CD8. Interestingly, we identify enhanced $\mathrm{CPG}$ methylation and mRNA expression of Runx3, a gene known to regulate $\mathrm{CD}^{+} / \mathrm{CD}^{+} \mathrm{T}$ lymphocyte development (21-24) by silencing $\mathrm{CD}^{+}$expression. The reduced level 
of Runx3 corresponds well with an increased $\mathrm{CD}^{+} / \mathrm{CD}^{+}$ratio. Run $x 3$ is a tumor suppressor gene (25) as well as a suppressor of dendritic cell maturation $(26,27)$, and it can regulate T lymphocyte development (21-23). Recent studies also suggest that Runx3 expression can be attenuated through increased DNA methylation $(28,29)$. Importantly, Runx3-deficient mice spontaneously develop an asthma-like phenotype (30). Recent findings indicate that Runx 3 cooperates with T-bet in the silencing of IL- 4 in the Th 1 cell (31). A reduced Runx3 level might lead to enhanced transcription of IL-4 skewing toward Th2 differentiation as observed in anti$\mathrm{CD}^{+}{ }^{+} \mathrm{CD} 28^{+}$antibody-stimulated $\mathrm{CD}^{+}$lymphocytes. In aggregate, our findings further support the importance of Runx3 as an epigenetically regulated candidate gene in allergic airway disease. Our study indicates that maternal dietary intake is an important early exposure that can directly influence $\mathrm{CpG}$ methylation, gene expression, and $\mathrm{T}$ lymphocyte development.

Consistent with an epigenetic mechanism that regulates the severity of allergic asthma in this model, we observed transgenerational inheritance of this phenotype. Previous work indicates that maternal diet consumption results in alterations in coat color in the agouti mouse as a result of differential $\mathrm{CpG}$ methylation $(7,8)$. Additionally, epigenetic modifications can be transgenerationally inherited through persistence of epigenetic changes in the germ line $(6,32,33)$. Our observation of partial transmission of disease risk suggests that the epigenetic effects on allergic airway disease are indeed reduced but persist into the F2 generation. Recently, dietary folic acid intake has been associated with CPG methylation in humans, which can be transmitted across generations (34). In addition to dietary exposures, it is likely that other environmental exposures could have an impact on epigenetic transmission of complex disease. For example, it is known that tobacco smoke can modify gene expression by DNA hypermethylation (35). Our discovery that the severity of allergic airway disease caused by in utero exposure to methyl donors can be passed through generations is consistent with an epigenetic mechanism of transmission. However, these transgeneration affects should be interpreted with caution, since the F2 generation germ line could have been exposed during gestation of the F1 generation (36). In aggregate, these observations support the complexity of heritable diseases and raise the possibility that epigenetic modification could contribute to the limited success of both linkage and association studies in complex heritable diseases such as asthma.

These observations suggest that the increased prevalence of allergic asthma in humans may in part be related to increased perinatal dietary supplementation with methyl donors. Given the importance of folate supplementation in preventing congenital abnormalities (3) and the differences in murine and human biology, we need to carefully evaluate the potential for adverse consequences of dietary supplementation during pregnancy before considering modifications in the current recommendations. However, our research suggests that too much dietary supplementation, especially with methyl donors during pregnancy, may have unexpected biological and pathophysiological consequences. Understanding the complex interactions between environmental exposures and genetic vulnerability will provide insight into future interventions for individuals at risk for the development of allergic asthma.

\section{Methods}

Methyl donor diet and breeding protocol. Methyl-supplemented diets were designed to provide an increase in the total amount of both cofactors and methyl donors required for methyl metabolism. AIN-93G (Research Diets Inc.) was supplemented during manufacturing with folic acid, vitamin B12, choline, L-methionine, zinc, and betaine. Choline is from choline chloride, betaine is anhydrous, and zinc is from $\mathrm{ZnSO}_{4} \bullet 7 \mathrm{H}_{2} \mathrm{O}(8)$. Genistein $(300 \mathrm{mg} /$ $\mathrm{kg}$ diet) was added as a source of phytoestrogens, which have been demonstrated to alter levels of DNA methylation (37). The supplemented amounts of all components in the HMD or LMD are reported in Supplemental Table 1. Each of the experimental diets (HMD, LMD) provided sufficient levels of vitamins to facilitate normal growth, development, and reproduction. Standard NIH-31 chow was considered RD (Harlan Teklad). C57BL/6J mice were treated with HMD, RD, or LMD for 2 weeks prior to mating with $\mathrm{C} 57 \mathrm{BL} / 6 \mathrm{~J}$ males and during pregnancy. The $\mathrm{F} 1$ progeny were placed on $\mathrm{RD}$ at the time of weaning (7). F2 progeny were generated by breeding the F1 males exposed to either HMD or LMD in utero with wild-type, untreated C57BL/6J females to generate F2 progeny. The F2 mice were kept on RD. The number of animals phenotyped in each generation included the following: $\mathrm{F} 1$ (HMD, 31; RD, 63; LMD, 34); F2 (HMD, 14; LMD, 19).

OVA sensitization and challenge. Eight- to twelve-week-old C57BL/6J mice (The Jackson Laboratory) were immunized and subjected to an OVA challenge as previously described (38). In brief, animals were immunized on days 0 and 7 by i.p. injections of $10 \mu \mathrm{g}$ of OVA (Sigma-Aldrich) complemented with $2.0 \mathrm{mg}$ of aluminum hydroxide (alum) (AlumInject; Pierce Biotechnologies Inc.) in a total volume of $100 \mu$ l. Control mice were sensitized by i.p. injection of $2.0 \mathrm{mg}$ alum alone in a total volume of $100 \mu \mathrm{l}$ on the same days. On days 14-16, mice were exposed to an aerosol of $1 \%$ OVA generated using a collision 6-jet nebulizer (BGI Inc.) for 40 minutes each day. Animals were phenotyped on day 17. Experimental protocols were reviewed and approved by the Institutional Animal Care and Use Committee at the National Institute of Environmental Health Sciences.

Airway physiology. Anesthesia was achieved with $60 \mathrm{mg} / \mathrm{kg}$ of pentobarbital sodium injected i.p. Mice were then given neuromuscular blockade $(0.8 \mathrm{ml} / \mathrm{kg}$ pancuronium bromide) and ventilated with a computer-controlled small animal ventilator (flexiVent; SCIREQ), with a tidal volume of $7.5 \mathrm{ml} / \mathrm{kg}$ and a positive end-expiratory pressure of $3 \mathrm{~cm} \mathrm{H}_{2} \mathrm{O}$. Measurements of respiratory mechanics were made by the forced oscillation technique. Response to aerosolized methacholine $(0,10 \mathrm{mg} / \mathrm{ml}, 25 \mathrm{mg} / \mathrm{ml}$, and $50 \mathrm{mg} / \mathrm{ml}$ ) was determined by resistance measurements every 30 seconds for 5 minutes, ensuring the parameters calculated had peaked. The lungs were inflated to total lung capacity after each dose of methacholine, maintaining open airways and returning the measurements back to baseline. The resistance measurements were then averaged at each dose and graphed $\left(\mathrm{R}_{T}\right.$, measured in $\mathrm{cmH}_{2} \mathrm{O} / \mathrm{ml} / \mathrm{s}$ ) along with the initial baseline measurement.

Whole-lung lavage. Whole-lung lavage was completed and cell differentials were determined, as previously described (38). Lavage cells were spun onto a glass slide using a cytocentrifuge (Cytospin 2; Shandon Southern) and stained using a Hemacolor (EMD). Cell-free lavage supernatants were stored at $-70^{\circ} \mathrm{C}$. Supernatants were concentrated for measurement of IL-13 using a Centricon YM-3 (Millipore) according to the manufacturer's instructions.

Cytokine and serum IgE. ELISA kit was used to evaluate concentration of IL-13 in whole-lung lavage fluid (R\&D Systems). Serum IgE levels were detected by ELISA (BD Biosciences - Pharmingen). Multiplexed fluorescent bead-based immunoassays (Bio-Rad) was used to evaluate concentration of 23 cytokines from supernatants from cultured splenocytes, thoracic lymph nodes, and thymocytes. Results are reported only for consistent significant differences in all 3 populations of lymphoid organs examined. ELISA kits and Bio-Plex suspension array system were used according to the manufacturer's instructions.

Western blot. Lung tissues from F1 progeny exposed to an in utero diet supplemented with methyl donors were homogenized and lysed in icecold lysis buffer containing $20 \mathrm{mM}$ Tris, $\mathrm{pH} 7.4,137 \mathrm{mM} \mathrm{NaCl}, 25 \mathrm{mM}$ 
$\beta$-glycerol phosphate, $\mathrm{pH}$ 7.4, 2 mM PPiNa, 2 mM EDTA, $\mathrm{pH}$ 7.4, 1\% Triton $\mathrm{X}-100,10 \%$ glycerol, $1 \mathrm{mM}$ PMSF, $5 \mu \mathrm{g} / \mathrm{ml}$ leupeptin, $5 \mu \mathrm{g} / \mathrm{ml}$ pepstatin, $5 \mu \mathrm{g} / \mathrm{ml}$ aprotinin, $1 \mathrm{mM} \mathrm{Na}_{3} \mathrm{VO}_{4}$, and $1 \mathrm{mM} \mathrm{DTT}$. After centrifugation, tissue extracts were separated by SDS-PAGE and transferred to a PVDF membrane. The membrane was incubated with blocking buffer containing $1 \times$ Tris-buffered saline, $0.1 \%$ Tween- 20 , and $5 \% \mathrm{w} / \mathrm{v}$ nonfat dry milk for 1 hour at room temperature and was immunoblotted overnight at $4{ }^{\circ} \mathrm{C}$ with polyclonal antibodies to human AML2-/Runx3 (Active Motif). $\beta$-actin served as a sample-loading control (Cell Signaling Technology Inc.). Membranes were incubated with the HRP-conjugated anti-rabbit antibody (Cell Signaling Technology Inc.) for 1 hour at room temperature. Protein was detected by the ECL Plus (Amersham Biosciences). Densitometric analysis was performed by ImageJ densitometry software (NIH) after scanning of the films. Runx3 protein levels were normalized to $\beta$-actin protein levels.

Lymphocyte assays. For flow cytometry, $1 \times 10^{6}$ cells from each animal were analyzed. Purified cells were blocked with Fc block, murine IgG, and rat IgG, then stained with fluorescent-conjugated monoclonal antibodies for CD3, CD4, and CD8 (BD Biosciences). Analysis was preformed using FACSVantage SE (BD Biosciences), and counts were calculated automatically by FlowJo software (TreeStar Inc.). Spleen, thoracic lymph nodes, and thymus tissue were harvested from F1 progeny of HMD or LMD mice after immunization and challenge with OVA. Cells were minced, passed through a $50-\mu \mathrm{m}$ cell strainer, and isolated by density gradient centrifugation with Histopaque 1083 (Sigma-Aldrich). To determine the functional implications of respective diets, $2 \times 10^{6}$ cells were cultured in RPMI with $10 \%$ FCS and exposed to $4 \mu \mathrm{g}$ OVA for 72 hours. Supernatants were collected and evaluated for cytokines/chemokines by Luminex protein profiling. Next, we harvested splenocytes from mice with gestational exposure to HMD or LMD without immunization/challenge to OVA and isolated CD4 ${ }^{+}$ lymphocytes by negative selection using MACS (Miltenyi Biotec). These cells were challenged with antibodies to $\mathrm{CD}^{+} \mathrm{CD}_{2} 8^{+}$(each $1 \mu \mathrm{g} / \mathrm{ml}$ ) for 48 hours. Supernatants were collected and analyzed by ELISA (BD Biosciences - Pharmingen) for levels of IL-4 and IFN- $\gamma$.

In vitro treatment with azacytidine. Splenocytes were isolated as above, cultured at concentrations of $1 \times 10^{6} \mathrm{cells} / \mathrm{ml}$, exposed to $4 \mu \mathrm{g}$ OVA in vitro for 72 hours, and pulsed daily with either saline or $1.0 \mu \mathrm{M}$ of azacytidine.

MSDK and data analysis. Genomic DNA was isolated from snap-frozen lung tissue samples that were subjected to an HMD or an LMD using a DNeasy kit (QIAGEN) according to the manufacturer's instructions. Two samples with low eosinophil counts (LMD) and 2 samples with high eosinophil counts (HMD) were selected for MSDK library construction. Library construction was essentially performed as described with a few minor modifications (18). For each library, $2 \mu \mathrm{g}$ of genomic DNA was digested with the mapping enzyme NotI, ligated to biotinylated NotI linkers (Integrated DNA Technologies), and digested with the fragmenting enzyme NlaIII. DNA fragments containing the biotinylated linkers were isolated using streptavidin-coated magnetic beads (Dynal Biotech) and ligated to linkers, including recognition sites for $\mathrm{MmeI}$. The 21-bp sequence tags were released by digestion with $\mathrm{MmeI}$ as has been described for Long SAGE (39). The isolated tags were self ligated, PCR amplified, concatenated, cloned in pZero (Invitrogen), and sequenced. DNA from plasmid inserts containing serial genomic tags was purified and sequenced at Agencourt Bioscience Corp. The SAGE 2000 software package enabled the extraction of the genomic tags from the sequence files. Tag sequences, tag counts, and gene associations were stored in a Microsoft Access relational database for subsequent selection and matching to virtual tags. The virtual genomic tags were extracted from the mouse genome sequence using a python script (http://www.python.org). This identified a total of 10,577 virtual tags, of which, 8,414 were unique. The mouse genome sequence was obtained from Ensembl (http://www.ensembl.org). A z-score was used to quantify the strength of the observed difference in the proportions of tags of a given sequence (count for the sequence divided by the total tag counts) in the 2 libraries.

Global DNA methylation. Measurement of the amount of methylation present in the mouse genome was performed using CpGlobal (Ambergen). In brief, 100 ng of genomic DNA was digested with HpaII and MspI (NEB) in triplicate in a 96 -well microtiter plate for 2 hours at $37^{\circ} \mathrm{C}$. The $5^{\prime}$ overhangs left after digestion were end filled with biotinylated nucleotides (PerkinElmer) for 30 minutes at $37^{\circ} \mathrm{C}$. The biotinylated DNA was bound to the surface of a 96-well MicroFluor 2 White Plate (Thermo Scientific) and left on a rotator overnight at room temperature. The wells were washed 4 times with wash solution, and the amount of biotin incorporated into the DNA was detected by HRP chemiluminescence (KPL). Luminescence was measured using a Wallac EnVision 2100 multilabel reader (PerkinElmer). The methylation index was calculated by dividing the values for HpaII luminescence by the average value for $M s p I$ luminescence.

Real-time PCR. Total RNA was extracted from frozen lung tissue using the RNAgents total RNA isolation system (Promega). Equal amounts of total RNA $(5 \mu \mathrm{g})$, as determined by the RiboGreen RNA quantification kit (Molecular Probes), were used in a $20 \mu \mathrm{l}$ cDNA synthesis reaction primed with oligo-dT (SuperScript II; Invitrogen). Control reactions were prepared in parallel without reverse transcriptase. Prior to cDNA synthesis, residual genomic DNA was removed from total RNA with a DNase I treatment (DNA-free; Ambion). Quantitative PCR was performed with a 7900HT Fast Real-Time PCR system (Applied Biosystems) using SYBR Green. PCR reactions were performed in triplicate, and the threshold cycle numbers were averaged. Gene expression levels were normalized to 3 genes: actin, beta (ACTB), $\beta$-2-microglobulin (B2M), and phosphoglycerate kinase 1 (PGK1). The relative expression levels were calculated in comparison with the levels in total RNA from naive mouse brain (Ambion) according to the comparative $\mathrm{Ct}$ method in which the relative expression equals $2^{-\Delta \Delta C t}$. PCR primers were designed using the Primer3 interface (http://www-genome.wi.mit. edu/cgi-bin/primer/primer3_www.cgi). A list of the PCR primers used for each gene follows: Runx3 forward, 5'-CAGGTTCAACGACCTTCGAT-3', Run $x 3$ reverse, 5'-AGGCCTTGGTCTGGTCTTCT-3'; Jak2 forward, 5'-TCTGTGGGAGATCTGCAGTG-3', Jak2 reverse, 5'-CACGGATGACAGCTCTGAAA-3'; Nfatc1 forward, 5'-TCATCCTGTCCAACACCAAA-3', Nfatc1, reverse $5^{\prime}$-TCACCCTGGTGTTCTTCCTC-3'; Rcor3 forward, 5'-CATGGATGGAAACGACAGTG-3', Rcor 3 reverse, $5^{\prime}$-AGTTGCCTCAGGATGGTGTT-3'; Actb forward, 5'-TCCGTAAAGACCTCTATGCC-3', Actb reverse, 5'-TACTCCTGCTTGCTGATCC-3'; and Ube2j1 forward, 5'-ACAGCAAGCACATCGTATGG-3', Ube2j1 reverse, 5'-ATAAGAGCCGCCAATGCTAA-3'.

Methylation-specific PCR and MALDI-TOF mass spectrometry. Sodium bisulfite modification of $500 \mathrm{ng}$ genomic mouse lung DNA was performed using the EZ DNA Methylation-Gold Kit (Zymo Research) according to the manufacturer's instructions. Modified DNA was eluted from the column with $20 \mu$ l elution buffer, aliquotted, and stored at $-20^{\circ} \mathrm{C}$. PCR reactions were performed as previously described, using $2 \mu \mathrm{l}$ modified genomic DNA (40). CPG islands at the $5^{\prime}$ region and up to the first exon of each gene were identified using the UCSC Genome Browser (http://genome. ucsc.edu/cgi-bin $/$ hgTrackUi?hgsid $=111780762 \& c=$ chr $4 \& g=$ cpgislandext $)$. The corresponding sequences were downloaded for primer design using MethPrimer (41) and Primer3. Primers were designed to contain at least 1 CpG dinucleotide, and multiple assays were established to evaluate various parts of a CpG island. Amplified products were analyzed by gel electrophoresis. Base-specific cleavage and MALDI-TOF mass spectrometry were performed by Sequenom Inc.

Statistics. Data were expressed as mean \pm SEM. Significant differences between groups were identified by analysis of variance. Individual comparisons between groups were confirmed by 2-tailed Student's $t$ test or the 
Mann-Whitney $U$ test in all cases except for the F2 phenotypes, where a 1 -tailed test was used because we were testing only 1 outcome, that an HMD was causing allergic airway inflammation in the F2 progeny. Statistical calculations were performed using SPSS (SPSS Inc.). $P<0.05$ was considered statistically significant.

\section{Acknowledgments}

This work was supported in part by the Intramural Research Program of the NIH, National Heart, Lung, and Blood Institute (to D.A. Schwartz). The authors also receive support through a National Heart, Lung, and Blood Institute career development award (HL91335 to J.W. Hollingsworth). We thank Richard Del Mastro for his support with the global DNA methylation assay and both Maria Sifre and John Whitesides for their assistance with flow cytometry. Histology analysis was provided by the Pathology
Support Group, a division of the Laboratory of Experimental Pathology, NIEHS. We appreciate the dedication from both Jessica Ramsberger and Sandy Hackney with animal husbandry.

Received for publication October 31, 2007, and accepted in revised form July 30, 2008.

Address correspondence to: John W. Hollingsworth, Division of Pulmonary, Allergy, and Critical Care Medicine, Duke University Medical Center, Box 3136, Durham, North Carolina 27710, USA. Phone: (919) 684-4588; Fax: (919) 684-3067; E-mail: holli017@mc.duke.edu.

John W. Hollingsworth, Shuichiro Maruoka, and Kathy Boon are co-first authors and contributed equally to this work.
1. Centers for Disease Control and Prevention (CDC). 2004. Asthma prevalence and control characteristics by race/ethnicity - United States, 2002. MMWR Morb. Mortal. Wkly. Rep. 53:145-148.

2. Eder, W., Ege, M.J., and von Mutius, E. 2006. The asthma epidemic. N. Engl. J. Med. 355:2226-2235.

3. Rosenquist, T.H., and Finnell, R.H. 2001. Genes, folate and homocysteine in embryonic development. Proc. Nutr. Soc. 60:53-61.

4. Moephuli, S.R., Klein, N.W., Baldwin, M.T., and Krider, H.M. 1997. Effects of methionine on the cytoplasmic distribution of actin and tubulin during neural tube closure in rat embryos. Proc. Natl. Acad. Sci. U. S. A. 94:543-548.

5. Feinberg, A.P., and Tycko, B. 2004. The history of cancer epigenetics. Nat. Rev. Cancer. 4:143-153.

6. Anway, M.D., Cupp, A.S., Uzumcu, M., and Skinner, M.K. 2005. Epigenetic transgenerational actions of endocrine disruptors and male fertility. Science. 308:1466-1469.

7. Waterland, R.A., and Jirtle, R.L. 2003. Transposable elements: targets for early nutritional effects on epigenetic gene regulation. Mol. Cell. Biol. 23:5293-5300.

8. Wolff, G.L., Kodell, R.L., Moore, S.R., and Cooney, C.A. 1998. Maternal epigenetics and methyl supplements affect agouti gene expression in Avy/a mice. FASEB J. 12:949-957.

9. Fields, P.E., Kim, S.T., and Flavell, R.A. 2002. Cutting edge: changes in histone acetylation at the IL-4 and IFN-gamma loci accompany Th1/Th2 differentiation. J. Immunol. 169:647-650.

10. Lee, G.R., Kim, S.T., Spilianakis, C.G., Fields, P.E., and Flavell, R.A. 2006. T helper cell differentiation: regulation by cis elements and epigenetics. Immunity. 24:369-379.

11. Shin, H.J., et al. 2005. STAT4 expression in human $\mathrm{T}$ cells is regulated by DNA methylation but not by promoter polymorphism. J. Immunol. 175:7143-7150.

12. Lee, D.U., Agarwal, S., and Rao, A. 2002. Th2 lineage commitment and efficient IL-4 production involves extended demethylation of the IL-4 gene. Immunity. 16:649-660.

13. Santangelo, S., Cousins, D.J., Winkelmann, N.E., and Staynov, D.Z. 2002. DNA methylation changes at human Th2 cytokine genes coincide with DNase I hypersensitive site formation during CD4(+) T cell differentiation. J. Immunol. 169:1893-1903.

14. Kim, H.P., and Leonard, W.J. 2007. CREB/ATFdependent $\mathrm{T}$ cell receptor-induced FoxP3 gene expression: a role for DNA methylation. J. Exp. Med 204:1543-1551.

15. Polansky, J.K., et al. 2008. DNA methylation controls Foxp3 gene expression. Eur. J. Immunol. 38:1654-1663.

16. Lukacs, N.W., et al. 1996. C-C chemokine-induced eosinophil chemotaxis during allergic airway inflammation. J. Leukoc. Biol. 60:573-578.

17. Fields, P.E., Lee, G.R., Kim, S.T., Bartsevich, V.V., and Flavell, R.A. 2004. Th2-specific chromatin remodeling and enhancer activity in the Th2 cytokine locus control region. Immunity. 21:865-876.

18. Hu, M., et al. 2005. Distinct epigenetic changes in the stromal cells of breast cancers. Nat. Genet. 37:899-905.

19. Wegmann, T.G., Lin, H., Guilbert, L., and Mosmann, T.R. 1993. Bidirectional cytokine interactions in the maternal-fetal relationship: is successful pregnancy a Th2 phenomenon? Immunol. Today. 14:353-356.

20. Holt, P.G., and Jones, C.A. 2000. The development of the immune system during pregnancy and early life. Allergy. 55:688-697.

21. Ehlers, M., et al. 2003. Morpholino antisense oligonucleotide-mediated gene knockdown during thymocyte development reveals role for Runx3 transcription factor in CD4 silencing during development of CD4-/CD8+ thymocytes. J. Immunol. 171:3594-3604.

22. Taniuchi, I., et al. 2002. Differential requirements for Runx proteins in CD4 repression and epigenetic silencing during $\mathrm{T}$ lymphocyte development. Cell. 111:621-633.

23. Woolf, E., et al. 2003. Runx3 and Runx1 are required for CD8 T cell development during thymopoiesis. Proc. Natl. Acad. Sci. U. S. A. 100:7731-7736.

24. Naoe, Y., et al. 2007. Repression of interleukin-4 in $\mathrm{T}$ helper type 1 cells by Runx/Cbf beta binding to the Il4 silencer. J. Exp. Med. 204:1749-1755.

25. Li, Q.L., et al. 2002. Causal relationship between the loss of RUNX3 expression and gastric cancer. Cell. 109:113-124.

26. Fainaru, O., et al. 2004. Runx3 regulates mouse TGF-beta-mediated dendritic cell function and its absence results in airway inflammation. EMBO J. 23:969-979.

27. Puig-Kroger, A., and Corbi, A. 2006. RUNX3: a new player in myeloid gene expression and immune response. J. Cell. Biochem. 98:744-756.

28. Mueller, W., et al. 2006. Downregulation of RUNX3 and TES by hypermethylation in glioblastoma.
Oncogene. 26:583-593.

29. Subramaniam, M.M., et al. 2008. RUNX3 inactivation by frequent promoter hypermethylation and protein mislocalization constitute an early event in breast cancer progression. Breast Cancer Res. Treat. Online publication ahead of print. doi:10.1007/ s10549-008-9917-4.

30. Fainaru, O., Shseyov, D., Hantisteanu, S., and Groner, Y. 2005. Accelerated chemokine receptor 7-mediated dendritic cell migration in Runx3 knockout mice and the spontaneous development of asthma-like disease. Proc. Natl. Acad. Sci. U. S. A. 102:10598-10603.

31. Djuretic, I.M., et al. 2007. Transcription factors T-bet and Runx3 cooperate to activate Ifng and silence Il 4 in T helper type 1 cells. Nat. Immunol. 8: $145-153$.

32. Morgan, H.D., Sutherland, H.G., Martin, D.I., and Whitelaw, E. 1999. Epigenetic inheritance at the agouti locus in the mouse. Nat. Genet. 23:314-318.

33. Rakyan, V.K., et al. 2003. Transgenerational inheritance of epigenetic states at the murine Axin $(\mathrm{Fu})$ allele occurs after maternal and paternal transmission. Proc. Natl. Acad. Sci. U. S. A. 100:2538-2543.

34. Jiang, Y.H., Bressler, J., and Beaudet, A.L. 2004. Epigenetics and human disease. Annu. Rev. Genomics Hum. Genet. 5:479-510.

35. Digel, W., and Lubbert, M. 2005. DNA methylation disturbances as novel therapeutic target in lung cancer: preclinical and clinical results. Crit. Rev. Oncol. Hematol. 55:1-11.

36. Skinner, M.K. 2008. What is an epigenetic transgenerational phenotype? F3 or F2. Reprod. Toxicol. 25:2-6.

37. Dolinoy, D.C., Weidman, J.R., Waterland, R.A., and Jirtle, R.L. 2006. Maternal genistein alters coat color and protects Avy mouse offspring from obesity by modifying the fetal epigenome. Environ. Health Perspect. 114:567-572.

38. Hollingsworth, J., et al. 2006. TLR4 signaling attenuates ongoing allergic inflammation. J. Immunol. 176:5856-5862.

39. Saha, S., et al. 2002. Using the transcriptome to annotate the genome. Nat. Biotechnol. 20:508-512.

40. Herman, J.G., Graff, J.R., Myohanen, S., Nelkin, B.D., and Baylin, S.B. 1996. Methylation-specific PCR: a novel PCR assay for methylation status of CPG islands. Proc. Natl. Acad. Sci. U. S. A. 93:9821-9826.

41. Li, L.C., and Dahiya, R. 2002. MethPrimer: designing primers for methylation PCRs. Bioinformatics. 18:1427-1431. 